\title{
Presynaptic Induction and Expression of Timing-Dependent Long-Term Depression Demonstrated by Compartment- Specific Photorelease of a Use-Dependent NMDA Receptor Antagonist
}

\author{
Antonio Rodríguez-Moreno, ${ }^{1,2,3 *}$ Michael M. Kohl, ${ }^{1,2 *}$ James E. Reeve, ${ }^{4}$ Thomas R. Eaton, ${ }^{4}$ Hazel A. Collins, ${ }^{4}$ \\ Harry L. Anderson, ${ }^{4}$ and Ole Paulsen ${ }^{1,2}$ \\ ${ }^{1}$ Department of Physiology, Development and Neuroscience, University of Cambridge, Cambridge CB2 3EG, United Kingdom, ${ }^{2}$ Department of Physiology, \\ Anatomy and Genetics, University of Oxford, Oxford OX1 3PT, United Kingdom, ${ }^{3}$ Department of Physiology, Anatomy and Cellular Biology, University \\ Pablo de Olavide, 41013 Seville, Spain, and ${ }^{4}$ Department of Chemistry, University of Oxford, Oxford OX1 3TA, United Kingdom
}

NMDA receptors are important for synaptic plasticity, including long-term potentiation (LTP) and long-term depression (LTD). To help investigate the precise location of the NMDA receptors that are required for different types of synaptic plasticity, we synthesized a caged form of the use-dependent NMDA receptor antagonist MK801, which we loaded into individual neurons in vitro, followed by compartment-specific uncaging. We used this method to investigate timing-dependent plasticity at layer 4-layer $2 / 3$ synapses of mouse barrel cortex. Somatodendritic photorelease of MK801 in the postsynaptic neuron produced a use-dependent block of synaptic NMDA receptor-mediated currents and prevented the induction of LTP. Compartment-specific photorelease of MK801 in the presynaptic neuron showed that axonal, but not somatodendritic, presynaptic NMDA receptors are required for induction of LTD. The rate of use-dependent block of postsynaptic NMDA receptor current was slower following induction of LTD, consistent with a presynaptic locus of expression. Thus, this new caged compound has demonstrated the axonal location of NMDA receptors required for induction and the presynaptic locus of expression of LTD at layer 4-layer 2/3 synapses.

\section{Introduction}

Spike timing-dependent plasticity (STDP) is a strong candidate for a synaptic mechanism involved in cortical development and map plasticity (Song and Abbott, 2001; Feldman and Brecht, 2005; Caporale and Dan, 2008). In STDP, the temporal order and millisecondprecision relative timing of presynaptic and postsynaptic action potentials (spikes) determine the direction and magnitude of synaptic change. Thus, long-term potentiation (LTP) occurs when a presynaptic spike is followed by a postsynaptic spike, whereas long-term depression (LTD) is induced when this order is reversed (Markram et al., 1997; Bi and Poo, 1998; Debanne et al., 1998; Caporale and Dan, 2008).

\footnotetext{
Received Jan. 17, 2011; revised April 22, 2011; accepted April 29, 2011.

Author contributions: A.R.-M., M.M.K., H.L.A., and 0.P. designed research; A.R.-M. and M.M.K. performed research; J.E.R., T.R.E., H.A.C., and H.L.A. contributed unpublished reagents/analytic tools; A.R.-M. and M.M.K. analyzed data; A.R.-M., M.M.K., and O.P. wrote the paper.

This work was supported by the Biotechnology and Biological Sciences Research Council, the Wellcome Trust OXION initiative, and by a Network grant from the MCDonnell Network for Cognitive Neuroscience, University of Oxford. This work was made possible by an Equipment Grant from the Alzheimer's Research Trust (funded by Doris Field (T).

${ }^{*}$ A.R.-M. and M.M.K. contributed equally to this work.

This article is freely available online through the J Neurosci Open Choice option.

Correspondence should be addressed to either of the following: Dr. Antonio Rodríguez-Moreno, Department of Physiology, Anatomy and Cellular Biology, University Pablo de Olavide, Carretera de Utrera, Kilometre 1 (Edificio 21), 41013 Seville, Spain, E-mail: arodmor@upo.es; or Ole Paulsen, Department of Physiology, Development and Neuroscience, University of Cambridge, Physiological Laboratory, Downing Street, Cambridge CB2 3EG, UK. E-mail: op210@cam.ac.uk.

DOI:10.1523/JNEUROSCI.0274-11.2011

Copyright $\odot 2011$ the authors $\quad 0270-6474 / 11 / 318564-06 \$ 15.00 / 0$
}

Both timing-dependent LTP (t-LTP) and LTD ( $t$-LTD) require NMDA receptors (NRs) (Bi and Poo, 1998; Debanne et al., 1998; Feldman, 2000; Sjöström et al., 2003; Bender et al., 2006; Nevian and Sakmann, 2006; Rodríguez-Moreno and Paulsen, 2008). Strong evidence shows that t-LTP requires postsynaptic NRs (Bi and Poo, 1998; Debanne et al., 1998; Feldman, 2000; Bender et al., 2006; Nevian and Sakmann, 2006), but the precise location of NRs mediating t-LTD remains controversial. Recent data suggest that t-LTD requires activation of presynaptic NRs at synapses on neocortical layer (L) 5 cells (Sjöström et al., 2003) and L2/3 cells (Nevian and Sakmann, 2006; Bender et al., 2006; Corlew et al., 2007; Rodríguez-Moreno and Paulsen, 2008). These presynaptic NRs are often assumed to be at axonal locations (Corlew et al., 2008). However, the existence of presynaptic, axonal NRs has been challenged by the discovery that somatodendritic NRs can affect axonal calcium channels in the same neuron (Christie and Jahr, 2008) and by the suggestion that NRs are excluded from the axon, at least in L5 neurons (Christie and Jahr, 2009). This raises the question of the exact location of those presynaptic NRs that are required for induction of t-LTD. To investigate this question directly, we developed a caged form of the intracellular NR channel blocker, MK801. We demonstrate that axonal, but not somatodendritic, photorelease of MK801 in presynaptic L4 neurons blocks the induction of t-LTD at L4-L2/3 synapses. Furthermore, we estimated changes in the probability of neurotransmitter release by measuring the trial-to-trial pro- 

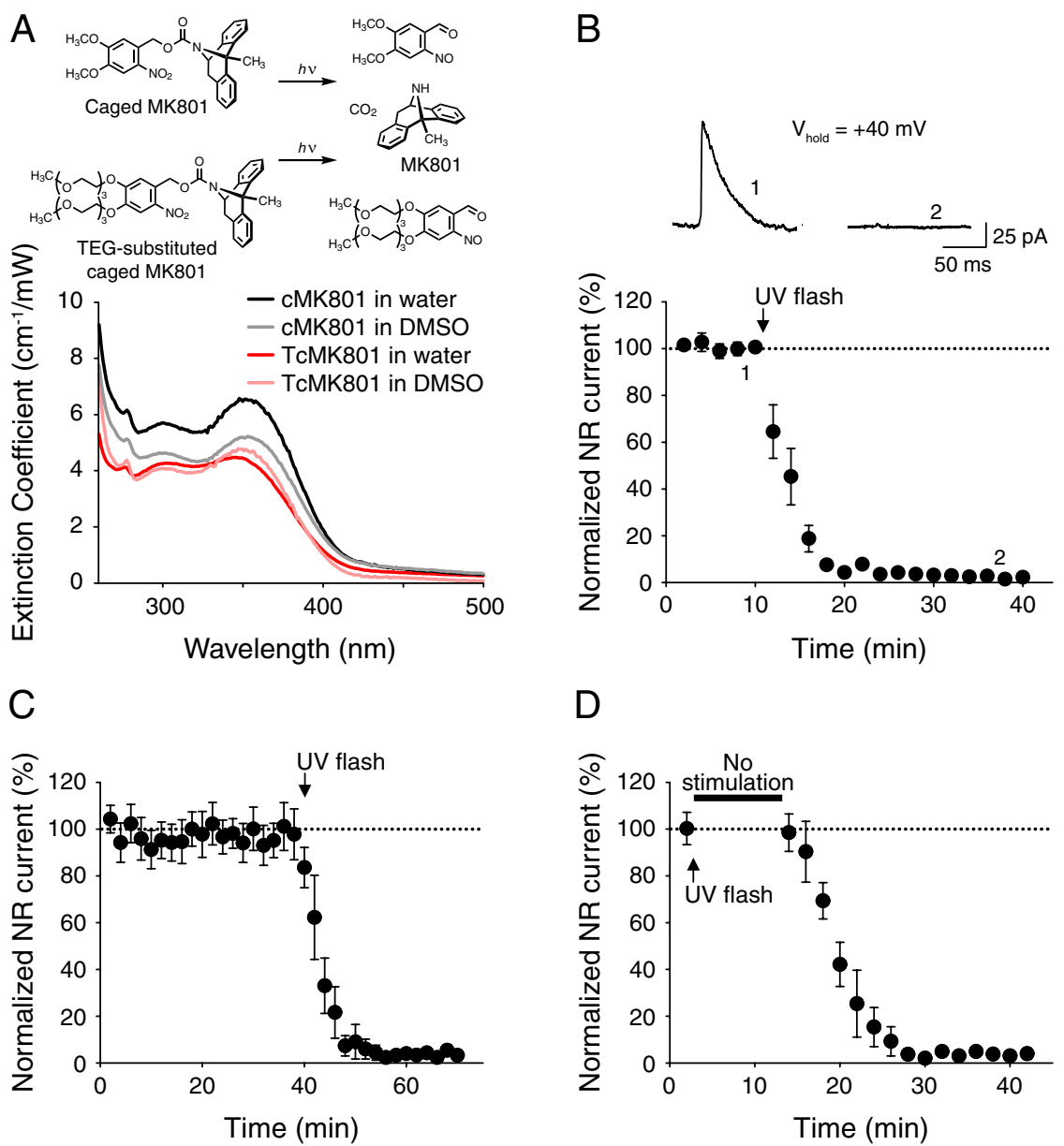

Figure 1. Properties of caged MK801. $\boldsymbol{A}$, Chemical structure, uncaging reaction, and absorption spectra of CMK801 and TCMK801. B, C, Effect of a UV flash on NR-EPSC in cMK801-loaded L2/3 neurons with flash after $10 \mathrm{~min}(\boldsymbol{B})$ or $40 \mathrm{~min}$ (C). Inset, NR-EPSC before (1) and 30 min after (2) UV flash. D, Use dependence of MK 801 block demonstrated by stopping stimulation for 10 min immediately after UV flash.

gressive decay of NR-mediated current amplitude following postsynaptic photorelease of MK801. We found that the rate of decay was slower following induction of t-LTD, consistent with a presynaptic locus of expression. We conclude that compartment-specific release of ion channel blockers is a powerful technique and have used this technique to demonstrate the precise location of NRs in synaptic plasticity.

\section{Materials and Methods}

Chemical synthesis. Caged MK801 (dizocilpine N-(4,5-dimethoxy-2nitrobenzyl) carbamate; cMK801) was prepared by adaptation of a published procedure (Frankel et al., 2003). Water solubility was increased by substituting this compound's two methoxy groups with triethylene glycol (TEG), yielding TEG-substituted caged MK801 (TcMK801) (Fig. 1A). Saturation concentration in pure $\mathrm{H}_{2} \mathrm{O}$ was as follows: cMK801, $0.2 \mu \mathrm{M}$; TcMK801, $9.8 \mu \mathrm{M}$. Stock solutions (100 mM) of both compounds were made up in DMSO and added to the pipette solution to yield a final concentration of $1 \mathrm{~mm}$.

Slice preparation. Slices from the barrel cortex were prepared from C57BL/6 mice of either sex (supplied by Harlan, UK), ranging from postnatal day (P) 9 to $\mathrm{P} 14$, as previously described (Rodríguez-Moreno and Paulsen, 2008). Briefly, mice were anesthetized with isoflurane and decapitated in accordance with UK Animals (Scientific Procedures) Act 1986. Slices were maintained at room temperature $\left(22-27^{\circ} \mathrm{C}\right)$ until used (1-8 h) in artificial CSF containing the following (in mM): $126 \mathrm{NaCl} ; 3 \mathrm{KCl}$; $1.25 \mathrm{NaH}_{2} \mathrm{PO}_{4} ; 2 \mathrm{MgSO}_{4}, 2 \mathrm{CaCl}_{2}, 26 \mathrm{NaHCO}_{3} ; 10$ glucose, pH 7.2-7.4; bubbled with carbogen gas $\left(95 \% \mathrm{O}_{2} / 5 \% \mathrm{CO}_{2}\right)$. All recordings were made at room temperature.

Recording conditions. Barrels were identified under a stereomicroscope. One or two stimulation electrodes were placed at the base of a barrel (L4). Whole-cell patch-clamp recordings were obtained from L2/3 pyramidal cells in the same barrel column with 5-7 $\mathrm{M} \Omega$ borosilicate pipettes. In voltage-clamp experiments, the pipette solution contained the following (in mM): $140 \mathrm{CsCl}$; 0.2 EGTA; 10.0 HEPES; 2.0 ATP-Mg; 0.3 GTP-NaCl; 5.0 QX-314, adjusted to $\mathrm{pH} 7.2$ with $\mathrm{CsOH}$. Voltage-clamp experiments were performed in the presence of gabazine $(2 \mu \mathrm{M})$ and NBQX $(10 \mu \mathrm{M})$. For current-clamp experiments, the pipette solution contained the following (in $\mathrm{mM}$ ): 110 potassium gluconate; 40 HEPES; 4 NaCl; 4 ATPMg; 0.3 GTP, adjusted to $\mathrm{pH} 7.2$ with $\mathrm{KOH}$. The pipette tip was filled with standard solution and backfilled with cMK801- or TcMK801-containing solution. Cells with a pyramidal-shaped soma were selected for recording using infrared, differential interference contrast optics. All cells were tested for regular spiking responses to positive current injection, characteristic of pyramidal cells. Whole-cell recordings were made using an Axon Multiclamp 700B amplifier (Molecular Devices). Recordings were low-pass filtered at $2 \mathrm{kHz}$ and acquired at $5 \mathrm{kHz}$ using an ITC-16 board (Instrutech) and custom-made software procedures programmed in Igor Pro (WaveMetrics). Cells were rejected if series resistance changed by $>15 \%$ during the recording.

MK801 uncaging. Photolysis of the caged MK801 was achieved with flashes of focused ultra-violet (UV) light (1 s, $\sim 120 \mu \mathrm{m}$ diameter, $<460 \mathrm{~nm}$ ) produced by a $100 \mathrm{~W}$ mercury arc lamp (HBO 100, Zeiss in combination with a Uniblitz T123 Driver and LS5 shutter system) connected to the epifluorescence port of the microscope (Axioskop, Zeiss) via a fiberoptic light-guide. Light intensity was $\sim 20 \mu \mathrm{W} / \mathrm{cm}^{2}$ after passing through a dichroic filter (FT460, Zeiss) and the $40 \times$ objective (Achroplan, 0.75 NA, Zeiss).

Timing-dependent LTP and LTD. EPSPs were evoked at $0.2 \mathrm{~Hz}$. After a stable EPSP baseline period of at least $10 \mathrm{~min}$, EPSPs were paired with single postsynaptic spikes (100 repetitions). To induce t-LTP, a prebefore-post pairing protocol was used in which the EPSP was followed after $\sim 10 \mathrm{~ms}$ by a single postsynaptic spike elicited by a brief depolarizing current pulse, whereas a post-before-pre pairing protocol in which a postsynaptic action potential was elicited $\sim 15 \mathrm{~ms}$ before the onset of the EPSP was used to induce t-LTD. The EPSP was monitored for at least 30 min after the end of the pairing period. Presynaptic stimulation frequency remained constant throughout the experiment. Plasticity was assessed from the slope of the EPSP, measured on the rising phase of the EPSP as a linear fit between time points corresponding to $25-30 \%$ and $70-75 \%$ of the peak amplitude during control conditions. For statistical comparisons, the mean EPSP slope was calculated from 60 consecutive sweeps immediately before the start of the pairing (baseline) and compared with 60 sweeps corresponding to $25-30 \mathrm{~min}$ after the pairing. t-LTP and t-LTD were assessed as the ratio of the EPSP slope after pairing to the EPSP slope during baseline.

Paired recordings. t-LTD was also studied using recordings between synaptically connected pairs of neurons. One patch pipette was positioned in a barrel (L4) and the other in L2/3 of the same barrel column. Once double whole-cell patch-clamp configuration was achieved, a brief current pulse was repeatedly applied to the presynaptic cell (L4) to check 
whether presynaptic action potentials could evoke EPSPs in the postsynaptic cell. Only pairs in which a spike in the L4 cell was followed by a monosynaptic EPSP in the postsynaptic L2/3 cell were used. t-LTD was induced by a post-before-pre pairing protocol in which a single postsynaptic spike was followed by a presynaptic spike after $\sim 15 \mathrm{~ms}$, as described previously (Rodríguez-Moreno and Paulsen, 2008).

Drugs. NBQX, D-AP5, MK801 (dizocilpine), and SR95531 (gabazine) were purchased from Tocris Bioscience and Ascent Scientific.

Data analysis. Data were analyzed using Igor Pro software (Wavemetrics). In Figure 4, single exponentials were fitted to the experimental data using Sigmaplot 11 (Systat Software Inc). Statistical comparisons were made using onesample or two-sample two-tailed Student's $t$ test as appropriate. $P$ values $<0.05$ were considered significant. Data are presented as mean \pm SEM.

\section{Results}

A caged form of the irreversible NR openchannel blocker MK801 was synthesized by adaptation of a previously used caging procedure (Frankel et al., 2003). The new compound (dizocilpine $N$-(4,5dimethoxy-2-nitrobenzyl) carbamate) absorbs UV light $(\lambda<400 \mathrm{~nm})$, and is cleaved by exposure to UV light, liberating MK801 (Fig. 1A). To test whether the caged compound is stable within cells and whether MK801 could be specifically photoreleased by UV flashes in a brain slice preparation, we loaded individual $\mathrm{L} 2 / 3$ neurons of the mouse barrel cortex with cMK801 (1 mM) and monitored the postsynaptic NR current before and following a UV flash (Fig. $1 B$ ). The synaptic NR current was monitored during voltage-clamp at $+40 \mathrm{mV}$ and was isolated by the addition of $2 \mu \mathrm{M}$ gabazine and $10 \mu \mathrm{M}$ NBQX to the superfusion fluid. The NR current was stable before the UV flash. However, after a $10 \mathrm{~min}$ baseline, a 1-s-long UV flash produced a gradual decrease of the amplitude of the NR current and an almost complete block within $8-10 \mathrm{~min}$ after delivery of the UV flash $(3 \pm 3 \%$ of baseline amplitude, $n=6$ ) (Fig. $1 B$ ). This finding suggests that MK801 was efficiently uncaged by a 1-s-long UV flash. The caged compound itself did not seem to have any effect on the NR currents, as the shape, amplitude, and time course of the responses continued to be typical of NR-mediated currents when the flash was not delivered. To ensure that the compound was stable over the duration of synaptic plasticity experiments, we repeated the experiment with $40 \mathrm{~min}$ baseline, which yielded equivalent results $(4 \pm 3 \%, n=5$ ) (Fig. $1 C$ ). The NR block following photorelease was use-dependent, because stopping synaptic stimulation for 10 min prevented the block of NR current, whereas a progressive block was again observed on resuming stimulation (Fig. 1D).

To assess the use of cMK801 in synaptic plasticity experiments, we first tested whether postsynaptic photorelease of MK801 could successfully block the induction of t-LTP. To this end, we monitored EPSPs evoked by extracellular stimulation in L4 during whole-cell recording of L2/3 pyramidal cells. LTP was induced by 100 pairings of an EPSP followed by a single postsyn-
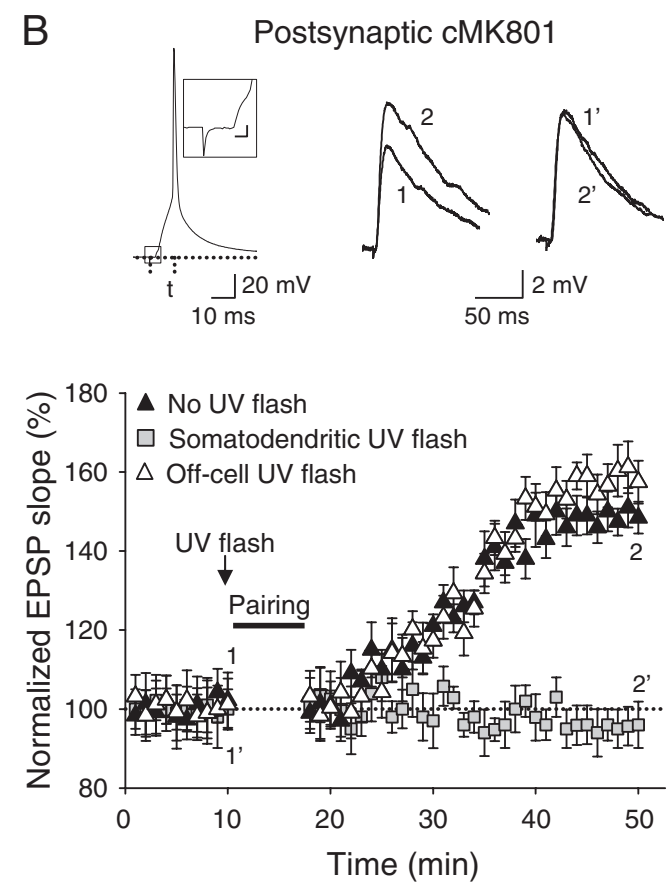

Figure 2. Photorelease of postsynaptic MK801 prevents spike timing-dependent LTP. $A$, Schematic of experimental setup. UV flash was delivered in somatodendritic area of postsynaptic neuron or off cell. $\boldsymbol{B}$, EPSP slopes monitored in cMK801-loaded cells in three experimental conditions: somatodendritic UV flash (gray squares), off-cell UV flash (white triangles), or no UV flash (black $\mathrm{mV}, 1 \mathrm{~ms}$. C, Summary of results. Error bars indicate SEM. ${ }^{* *} p<0.01$, Student's $t$ test. The number of slices used for each protocol is indicated in parentheses at the top of each error bar.

aptic spike (with the postsynaptic spike occurring $\sim 10 \mathrm{~ms}$ after presynaptic stimulation). When L2/3 cells were loaded with $1 \mathrm{~mm}$ cMK801, but with no flash applied, this pre-before-post pairing protocol induced robust t-LTP $(148 \pm 4 \%, n=5$; $p<0.01, t$ test $)$ (Fig. 2), similar to unloaded cells (Rodríguez-Moreno and Paulsen, 2008). In the same experimental condition, but with a somatodendritic UV flash delivered, the induction of t-LTP was completely blocked ( $96 \pm 6 \%, n=6$ ) (Fig. 2). To ascertain that the effect of the UV flash was specific to the cell loaded with cMK801, we delivered UV flashes $200 \mu \mathrm{m}$ lateral to the recording pipette. In this situation, t-LTP was unaffected $(157 \pm 6 \%, n=6)$ (Fig. 2). These results indicate that postsynaptic somatodendritic NRs are necessary for t-LTP induction consistent with previous results (Bender et al., 2006; Rodríguez-Moreno and Paulsen, 2008).

In contrast to the postsynaptic NRs involved in t-LTP, we have previously reported that $t-L T D$ requires presynaptic NRs (Rodríguez-Moreno and Paulsen, 2008). To investigate whether the NRs that are required for t-LTD are located in the axonal or the somatodendritic compartment of the presynaptic L4 neurons, we used paired whole-cell recordings of synaptically connected L4 and L2/3 cells as previously described (Rodríguez-Moreno and Paulsen, 2008). Of 332 pairs recorded, 31 pairs showed a monosynaptic EPSP (latency, $1.9 \pm 0.4 \mathrm{~ms}$; jitter, $0.12 \pm 0.04 \mathrm{~ms}$ ), 28 of which were used in plasticity experiments. First, a t-LTD induction protocol was applied to 12 pairs in which the presynaptic L4 neuron was loaded with $1 \mathrm{~mm}$ cMK801 (Fig. 3A). A UV flash confined to the somatodendritic region of the L4 neuron delivered $1 \mathrm{~min}$ before the start of the pairing protocol did not affect 
A

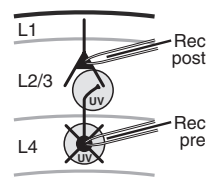

B

Presynaptic cMK801

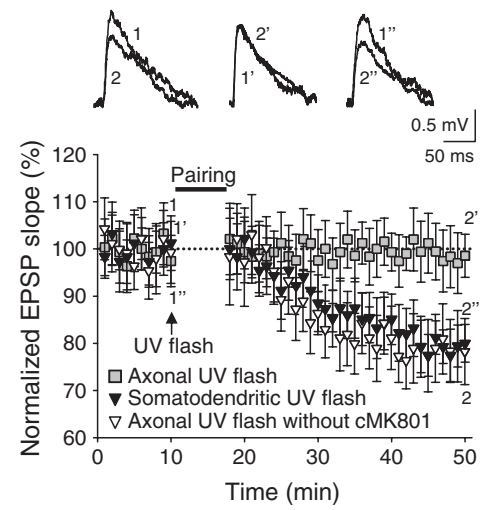

C

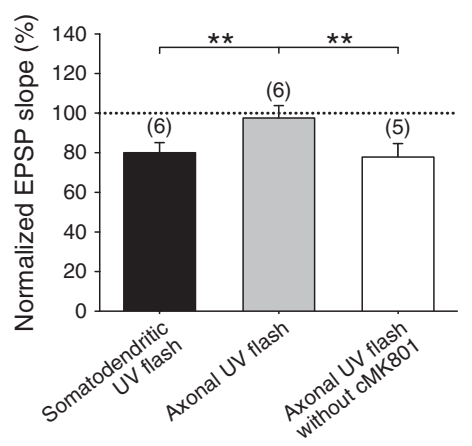

D

Axonal UV flash

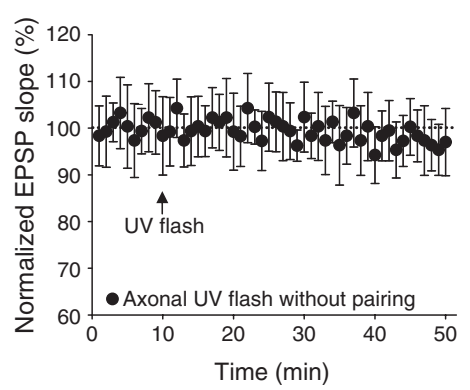

E Somatodendritic UV flash

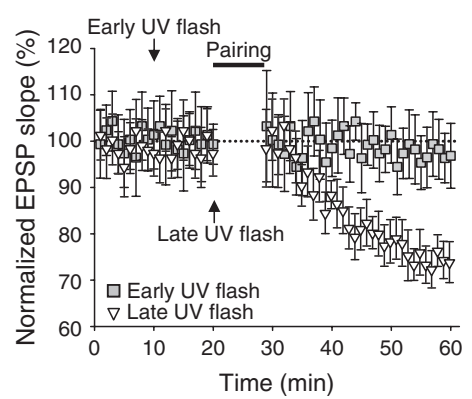

F

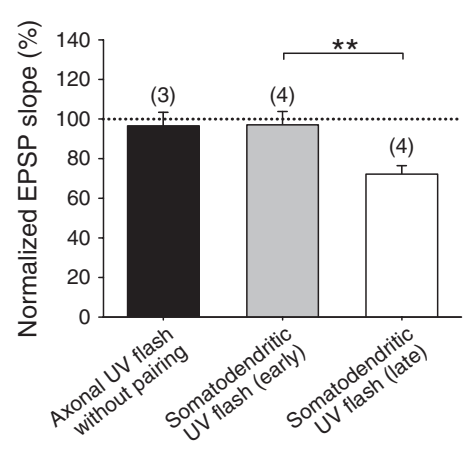

Figure 3. Spike timing-dependent LTD requires axonal NMDA receptors. $A$, Schematic of experimental setup. UV flash was delivered in either the somatodendritic or axonal region of the presynaptic neuron. $\boldsymbol{B}$, EPSP slopes monitored in pairs of cells in three experimental conditions: axonal UV flash on cMK801-loaded presynaptic cell (gray squares), somatodendritic UV flash on cMK801-loaded presynaptic cell (black triangles), or axonal UV flash on presynaptic cells not loaded with cMK801 (white triangles). Only axonal UV flash in the presence of presynaptic cMK801 blocked t-LTD. Inset, EPSP before $\left(1,1^{\prime}, 1^{\prime \prime}\right)$ and 30 min after t-LTD induction protocol $\left(2,2^{\prime}, 2^{\prime \prime}\right)$ with somatodendritic UV flash $(1,2)$, axonal UV flash with cMK801 $\left(1^{\prime}, 2^{\prime}\right)$, and axonal UV flash without CMK801 $\left(1, " 2^{\prime \prime}\right)$. C, Summary of results. Error bars indicate SEM. ${ }^{* *} p<0.01$, Student's $t$ test. The number of slices used for each protocol is indicated in parentheses at the top of each error bar. D, No effect of axonal UV flash when not followed by pairing. E, Effective block of t-LTD by presynaptic somatodendritic UV flash when applied 10 min before pairing (early UV flash; gray squares), compared to immediately before pairing (late UV flash; white triangles). $\boldsymbol{F}$, Summary of results. Error bars indicate SEM. ${ }^{* *} p<0.01$, Student's $t$ test. The number of slices used for each protocol is indicated in parentheses at the top of each error bar.

the induction of t-LTD $(80 \pm 5 \%, n=6 ; p<0.01, t$ test) (Fig. $3 B, C)$. In contrast, when the UV flash was delivered to the axonal region of the L4 cell (i.e., in layer 2/3) 1 min before the t-LTD induction protocol, t-LTD was completely blocked ( $97 \pm 4 \%$, $n=6)$ (Fig. $3 B, C)$. Axonal flash without pairing had no effect on the EPSP $(97 \pm 7 \%, n=3)$ (Fig. $3 D, F)$. To confirm that the block of t-LTD induction in the axonal region of L4 cells is mediated specifically by uncaging of MK801 and not by the UV flash itself, we repeated the experiment with no cMK801 included in the presynaptic pipette. In this condition, an axonal UV flash delivered 1 min before the t-LTD induction protocol did not affect t-LTD $(78 \pm 5 \%, n=5)($ Fig. $3 B, C)$. Moreover, if a 10 min delay was introduced between presynaptic somatodendritic uncaging and the pairing protocol, t-LTD was blocked $(96 \pm 7 \%, n=4)$
(Fig. $3 E, F$ ), suggesting that once photoreleased, free MK801 can diffuse into the axon and block the induction of t-LTD. Together, these results indicate that presynaptic axonal NMDA receptors are required for the induction of t-LTD.

Based on trial-to-trial fluctuation analysis (Rodríguez-Moreno and Paulsen, 2008), as well as changes in paired-pulse ratio (Bender et al., 2006), t-LTD at L4-L2/3 synapses has been proposed to be expressed presynaptically through a decrease in presynaptic release probability. Changes in release probability at excitatory synapses can be assessed by analyzing the progressive block of NR-mediated currents by MK801 (Hessler et al., 1993; Rosenmund et al., 1993). As MK801 is an irreversible NR open-channel blocker (Jahr, 1992), NRs are blocked only at synapses that release transmitter, so the trial-to-trial rate of block provides a measure of the release probability (Rosenmund et al., 1993). We wanted to take advantage of caged MK801 to investigate whether a change in release probability can account for the magnitude of synaptic depression. To this end, we used an improved, water-soluble version of cMK801 (TEG-substituted cMK801, TcMK801), which we loaded postsynaptically and monitored EPSPs evoked by extracellular stimulation in L4 during current-clamp wholecell recording of L2/3 pyramidal cells. t-LTD was induced in one pathway by 100 pairings of a postsynaptic action potential followed after $\sim 15 \mathrm{~ms}$ by an EPSP. This post-beforepre pairing protocol induced robust t-LTD (75 $\pm 6 \%, p<0.01, n=7$ ) (Fig. $4 A$ ). An unpaired naive pathway was used as control, and no changes in EPSP slopes were observed in that pathway $(101 \pm 6 \%, n=7)$ (Fig. 4A). Thirty to $35 \mathrm{~min}$ after the pairing protocol, we switched to voltage-clamp mode and recorded NR-EPSCs at $-30 \mathrm{mV}$ in the same cells. NR currents were isolated by the addition of gabazine $(2 \mu \mathrm{M})$ and NBQX $(10 \mu \mathrm{M})$ to the superfusion solution to block $\mathrm{GABA}_{\mathrm{A}}$ and $\mathrm{AMPA} /$ kainate receptors, respectively. After 10 min baseline, a 1-s-long UV flash was applied to photorelease MK801. After delivery of the UV flash, a gradual decrease of the amplitude of the NR currents and eventually an almost complete block was observed in the paired $(96 \pm 3 \%, n=7)$ as well as the unpaired pathway $(97 \pm 3 \%$, $n=7$ ) (Fig. 4B). However, a slower rate of decay (measured as the number of stimuli necessary to reduce NR-EPSC amplitude) was observed in the paired compared with the unpaired pathway (Fig. $4 B)$. The decay could be adequately fitted with a single exponential function $\left(R^{2}>0.90\right.$ in all inputs; $\left.n=14\right)$ (Fig. $4 B$ ). The half-life of the NR-EPSC was $32 \pm 4$ stimuli for the unpaired pathway $(n=7)$ and $46 \pm 4$ stimuli for the paired pathway $(n=7 ; p<0.05, t$ test $)$ (Fig. 4B). These results are consistent with a reduction in the neurotransmitter release probability in the paired pathway. To estimate the relative change in release probability, we compared the difference 
in reduction of estimated NR current after one stimulation between the paired and unpaired pathways. There was a $27 \pm 10 \%$ $(n=7)$ difference between the two pathways, which compares to $25 \pm 6 \%$ depression in the paired pathway $(n=7)$. This result suggests that a decrease in release probability can account for t-LTD, indicating a presynaptic locus of expression for this form of t-LTD.

\section{Discussion}

We have developed a caged form of MK801 to study synaptic plasticity. Our data confirm that postsynaptic NRs are necessary for t-LTP at L4-L2/3 synapses, induced by pairing single presynaptic and postsynaptic action potentials, which is an effective protocol for induction of t-LTP in developing cortex including hippocampus (Bi and Poo, 1998; Debanne et al., 1998; Meredith et al., 2003; Campanac and Debanne, 2008). In contrast, our results indicate that presynaptic axonal NMDA receptors are required for t-LTD at this synapse, as a spatially localized flash in the axonal but not the somatodendritic region of cMK801-loaded presynaptic cells blocked the induction of t-LTD. These results provide further evidence for the presence of functional NRs in the presynaptic axonal membrane (Berretta and Jones, 1996; Brasier and Feldman, 2008), supporting previous anatomical evidence for presynaptic NRs in the neocortex (Aoki et al., 1994; DeBiasi et al., 1996; Charton et al., 1999; Corlew et al., 2007).

The requirement of axonal NRs for induction of t-LTD raises the question of the source of ligand that activates these presynaptic NRs. The ligand(s) might be released by the postsynaptic neurons, as retrograde release of glutamate has been reported (Harkany et al., 2004) or from glial cells (Perea et al., 2009). Another possible source of ligand(s) could be the spill-over from neighboring synapses, although this appears less likely in paired recordings. Further experiments are needed to uncover the source of ligand(s) activating the axonal NRs required for t-LTD.

The use-dependent nature of NR block by MK801 (Jahr, 1992; Hessler et al., 1993; Rosenmund et al., 1993) enabled us to use uncaging of MK801 in the postsynaptic neuron to study the locus of expression of t-LTD. Our data provide evidence that MK801 produces a use-dependent block of NRs also when applied intracellularly. A slower rate of block in the depressed pathway compared with a naïve pathway suggests that t-LTD is expressed by reduced transmitter release probability, which is consistent with increased paired-pulse ratio (Bender et al., 2006) and coefficient of variation (Rodríguez-Moreno and Paulsen, 2008) following induction of t-LTD at this synapse.

In conclusion, we have successfully used intracellular compartment-specific photorelease of a channel blocker to study the locus of induction and expression of t-LTD, demonstrating that presynaptic axonal NRs are necessary for this presynaptically expressed LTD. Our results also confirm that t-LTP at the L4-to-L2/3 synapse in the barrel cortex is mediated by postsynaptic (L2/3) somatodendritic NRs. Thus, spatially and temporally precise uncaging of ion channel blockers is a useful tool to uncover compartment-specific biological functions.

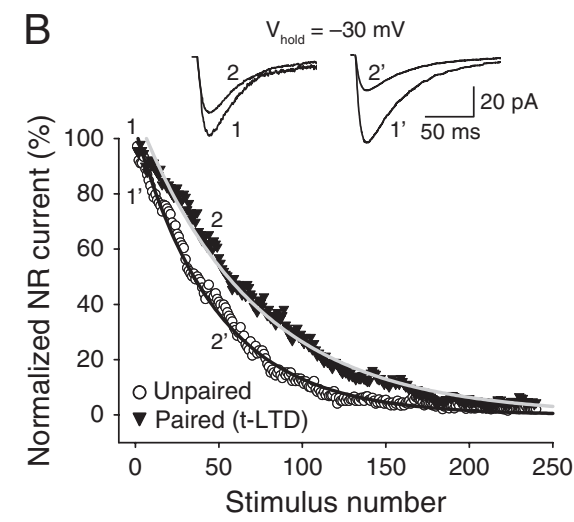

Figure 4. Spike timing-dependent LTD is expressed presynaptically. A, EPSP slopes monitored in paired (black triangles) and unpaired pathway (white circles). Traces show EPSP before (1) and 30 min after (2) t-LTD induction protocol in a paired pathway and at the equivalent times in the unpaired pathway $\left(1^{\prime}, 2^{\prime}\right)$. Only the paired pathway showed t-LTD. $B$, NR-EPSC amplitudes ved in the paired (black triangles) compared with the unpaired pathway (white circles). Traces show NR-EPSC just after

\section{References}

Aoki C, Venkatesan C, Go CG, Mong JA, Dawson TM (1994) Cellular and subcellular localization of NMDA-R1 subunit inmunorreactivity in the visual cortex of adult and neonatal rats. J Neurosci 14:5202-5222.

Bender VA, Bender KJ, Brasier DJ, Feldman DE (2006) Two coincidence detectors for spike timing-dependent plasticity in somatosensory cortex. J Neurosci 26:4166-4177.

Berretta N, Jones RS (1996) Tonic facilitation of glutamate release by presynaptic N-methyl-D-aspartate autoreceptors in the entorhinal cortex. Neuroscience 75:339-344.

Bi GQ, Poo MM (1998) Synaptic modifications in cultured hippocampal neurons: dependence on spike timing, synaptic strength, and postsynaptic cell type. J Neurosci 18:10464-10472.

Brasier DJ, Feldman DE (2008) Synapse-specific expression of functional presynaptic NMDA receptors in rat somatosensory cortex. J Neurosci 28:2199-2211.

Campanac E, Debanne D (2008) Spike timing-dependent plasticity: a learning rule for dendritic integration in rat CA1 pyramidal neurons. J Physiol 586:779-793.

Caporale N, Dan Y (2008) Spike timing-dependent plasticity: a Hebbian learning rule. Annu Rev Neurosci 31:25-46.

Charton JP, Herkert M, Becker CM, Schröder H (1999) Cellular and subcellular localization of the $2 \mathrm{~B}$-subunit of the NMDA receptor in the adult rat telencephalon. Brain Res 816:609-617.

Christie JM, Jahr CE (2008) Dendritic NMDA receptors activate axonal calcium channels. Neuron 60:298-307.

Christie JM, Jahr CE (2009) Selective expression of ligand-gated ion channels in L5 pyramidal cell axons. J Neurosci 29:11441-11450.

Corlew R, Wang Y, Ghermazien H, Erisir A, Philpot BD (2007) Developmental switch in the contribution of presynaptic and postsynaptic NMDA receptors to long-term depression. J Neurosci 27:9835-9845.

Corlew R, Brasier DJ, Feldman DE, Philpot BD (2008) Presynaptic NMDA receptors: newly appreciated roles in cortical synaptic function and plasticity. Neuroscientist 14:609-625.

Debanne D, Gähwiler BH, Thompson SM (1998) Long-term synaptic plasticity between pairs of individual CA3 pyramidal cells in rat hippocampal slice cultures. J Physiol 507:237-247.

DeBiasi S, Minelli A, Melone M, Conti F (1996) Presynaptic NMDA receptors in the neocortex are both auto- and heteroreceptors. Neuroreport 7:2773-2776.

Feldman DE (2000) Timing-based LTP and LTD at vertical inputs to layer II/III pyramidal cells in rat barrel cortex. Neuron 27:45-56.

Feldman DE, Brecht M (2005) Map plasticity in somatosensory cortex. Science 310:810-815.

Frankel A, Millward SW, Roberts RW (2003) Encodamers: unnatural peptide oligomers encoded in RNA. Chem Biol 10:1043-1050. 
Harkany T, Holmgren C, Härtig W, Qureshi T, Chaudhry FA, StormMathisen J, Dobszay MB, Berghuis P, Schulte G, Sousa KM, Fremeau RT Jr, Edwards RH, Mackie K, Ernfors P, Zilberter Y (2004) Endocannabinoid-independent retrograde signaling at inhibitory synapses in layer $2 / 3$ of neocortex: involvement of vesicular glutamate transporter 3. J Neuroci 24:4978-4988.

Hessler NA, Shirke AM, Malinow R (1993) The probability of transmitter release at a mammalian central synapse. Nature 366:569-572.

Jahr CE (1992) High probability opening of NMDA receptor channels by L-glutamate. Science 255:470-472.

Markram H, Lübke J, Frotscher M, Sakmann B (1997) Regulation of synaptic efficacy by coincidence of postsynaptic APs and EPSPs. Science 275:213-215.

Meredith RM, Floyer-Lea AM, Paulsen O (2003) Maturation of long-term potentiation induction rules in rodent hippocampus: role of GABAergic inhibition. J Neurosci 23:11142-11146.
Nevian T, Sakmann B (2006) Spine $\mathrm{Ca}^{2+}$ signaling in spike-timingdependent plasticity. J Neurosci 43:11001-11013.

Perea G, Navarrete M, Araque A (2009) Tripartite synapses, astrocytes process and control synaptic information. Trends Neurosci 32:421-431.

Rodríguez-Moreno A, Paulsen O (2008) Spike timing-dependent longterm depression requires presynaptic NMDA receptors. Nat Neurosci 11:744-745.

Rosenmund C, Clements JD, Westbrook GL (1993) Nonuniform probability of glutamate release at a hippocampal synapse. Science 262:754-757

Sjöström PJ, Turrigiano GG, Nelson SB (2003) Neocortical LTD via coincident activation of presynaptic NMDA and cannabinoid receptors. Neuron 39:641-654.

Song S, Abbott LF (2001) Cortical development and remapping through spike timing-dependent plasticity. Neuron 32:339-350. 